\title{
Primary pulmonary MALT lymphoma presenting as non-resolving pneumonia
}

The authors declare no financial disclosure

\begin{abstract}
Primary lung lymphoma (PLL) is a rare unilateral or bilateral lung disorder that is challenging to diagnose solely based on clinical and radiological presentation. PLL may be misdiagnosed as pneumonia or a lung tumor due to non-specific findings. PLL is most frequently a mucosa-associated lymphoid tissue (MALT) lymphoma, a type of extranodal low-grade B-cell lymphoma most commonly discovered in the gastrointestinal tract. PLL should be considered in the differential diagnosis of non-resolving pneumonias. Herein we present a case of an 84-year-old patient discovered to have a primary pulmonary MALT lymphoma presenting as a non-resolving pneumonia causing a clinical challenge.
\end{abstract}

Key words: primary pulmonary lymphoma, MALT lymphoma, non resolving pneumonia, lung cancer

Adv. Respir. Med. 2017; 85: 202-205

\section{Introduction}

Primary lung lymphoma (PLL) is a rare disease that encompasses many histologic types of lymphomas. It accounts for $<1 \%$ of all pulmonary neoplasms and only $3-4 \%$ of all extranodal non -Hodgkin's lymphomas (NHLs) with a median age of diagnosis in the sixth decade of life [1,2]. The most common histological type of PLL are those arising from mucosa-associated lymphoid tissue (MALT), which were first described by Isaacson and Wright in 1983 [2, 3]. Although MALT most frequently develops in the gastrointestinal tract, other sites can include the salivary glands, orbits, and lungs. MALT lymphoma may be associated with chronic inflammatory conditions and has been seen with chronic infections as well as autoimmune disorders e.g. Sjögren's Syndrome [2]. PLL is often asymptomatic; however, patients may present with non-specific respiratory symptoms such as cough, shortness of breath, or constitutional symptoms. Herein we describe a case of primary pulmonary MALT lymphoma presenting as a non-resolving pneumonia that caused a diagnostic challenge.

\section{Case report}

An 84-year-old, African American, non-smoker male with a history of hypertension (HTN) and benign prostatic hyperplasia (BPH) presented to his primary care physician with a two-week history of non-productive cough. He denied chest pain or shortness of breath. The patient was treated for possible walking pneumonia on an outpatient basis with doxycycline without resolution of his symptoms. Patient was then referred to a pulmonologist for further evaluation. He was retreated with levofloxacin and, again, failed to show improvement. He was then recommended for an

Address for correspondence: Asma Iftikhar, New York Presbyterian Hospital Queens Department of Pulmonary, Critical Care and Sleep Medicine 56-45 Main Street.

Flushing, NY 11355, e-mail: doctorasmi@gmail.com

DOI: 10.5603/ARM.2017.0033

Received: 8.01.2017

Copyright (C) 2017 PTChP

ISSN 2451-4934 


\section{Table 1. Initial laboratory findings}

\begin{tabular}{lc}
\hline WBC & $7.35(4.80-10.80 \mathrm{~K} / \mathrm{ul})$ \\
RBC & $4.46(4.50-5.90 \mathrm{M} / \mathrm{ul})$ \\
HGB & $12.5(13.5-17.5 \mathrm{~g} / \mathrm{dL})$ \\
HCT & $38.2(42-52 \%)$ \\
PLT & $398(150-400 \mathrm{~K} / \mathrm{ul})$ \\
MCV & $85.7(80-100 \mathrm{fL})$ \\
ESR & $61(0-25 \mathrm{~mm})$ \\
Glucose & $82(74-99 \mathrm{mg} / \mathrm{dL})$ \\
BUN & $29.0(8.0-23.0 \mathrm{mg} / \mathrm{dL})$ \\
Creatinine & $2.14(0.70-1.20 \mathrm{mg} / \mathrm{dL})$ \\
Sodium & $134(136-145 \mathrm{mmol} / \mathrm{L})$ \\
Potassium & $4.9(3.5-5.1 \mathrm{mmol} / \mathrm{L})$ \\
Chloride & $98(98-107 \mathrm{mmol} /)$ \\
Bicarbonate & $24(22-29 \mathrm{mmol} / \mathrm{l})$ \\
Calcium & $10.0(8.6-10.4 \mathrm{mg} / \mathrm{dL})$ \\
\hline Liver function tests & \\
\hline Protein (total) & $6.6(6.4-8.3 \mathrm{~g} / \mathrm{dl})$ \\
Albumin & $3.8(3.55 .2 \mathrm{~g} / \mathrm{dl})$ \\
AST & $17(5-40 \mathrm{U} / \mathrm{L})$ \\
ALT & $16(5-41 \mathrm{U} / \mathrm{L})$ \\
Alkaline phosphatase & $81(40-130 \mathrm{U} / \mathrm{L})$ \\
Bilirubin (total) & $0.5(0.0-1.2 \mathrm{mg} / \mathrm{dl})$ \\
Bilirubin (direct) & $0.1(0.0-0.9 \mathrm{mg} / \mathrm{dl})$ \\
Bilirubin (indirect) & $0.4(0.0-0.9 \mathrm{mg} / \mathrm{dl})$ \\
\hline & \\
\hline
\end{tabular}

outpatient bronchoscopy to rule out other possible infectious or rheumatological etiology to his symptoms, but the patient refused. His symptoms continued to persist and worsen with significant weight loss of nine kilograms over the course of a year and a half. Due to worsening cough the patient was sent to the emergency room for expeditious workup. Past medical history revealed a previous episode of pneumonia successfully treated with antibiotics approximately 18 months prior. The patient reported that he was a pharmacist, and denied a history of drug or alcohol use or exposure to any toxins. There was no known exposure to Mycobacterium tuberculosis or other communicable diseases. On examination, patient was afebrile with stable vital signs, blood pressure (BP) of $137 / 77 \mathrm{~mm} \mathrm{Hg}$, heart rate (HR) of 66 , oxygen saturation $\left(\mathrm{O} 2_{\text {sat }}\right.$ of $93 \%$, respiratory rate (RR) of 16 breaths per minute and a temperature of 36.5 degrees Celsius. Initial laboratory findings and liver function tests have been summarized in Table 1. Lactose dehydrogenase was not me-

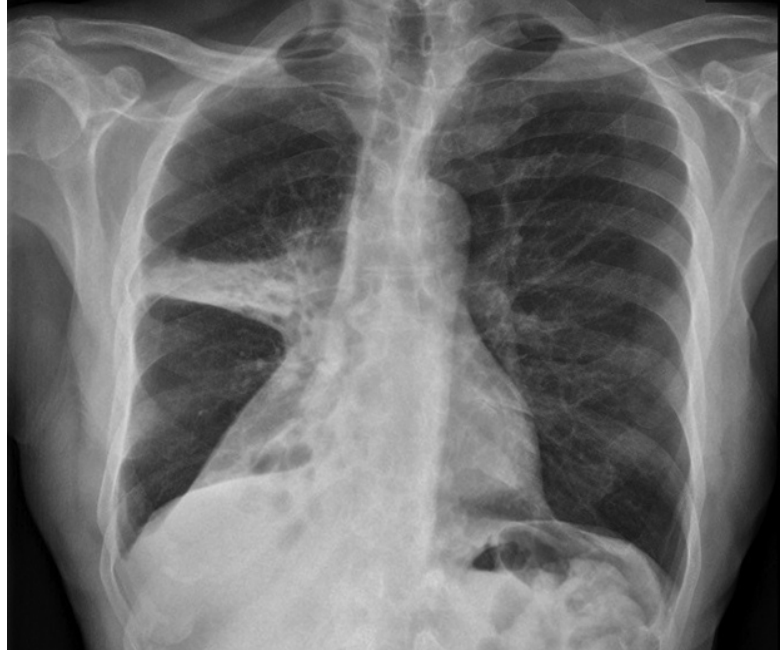

Figure 1. Chest X-ray Right upper and middle lobe scarring and atelectasis with cystic changes. Possible raised right hemi-diaphragm

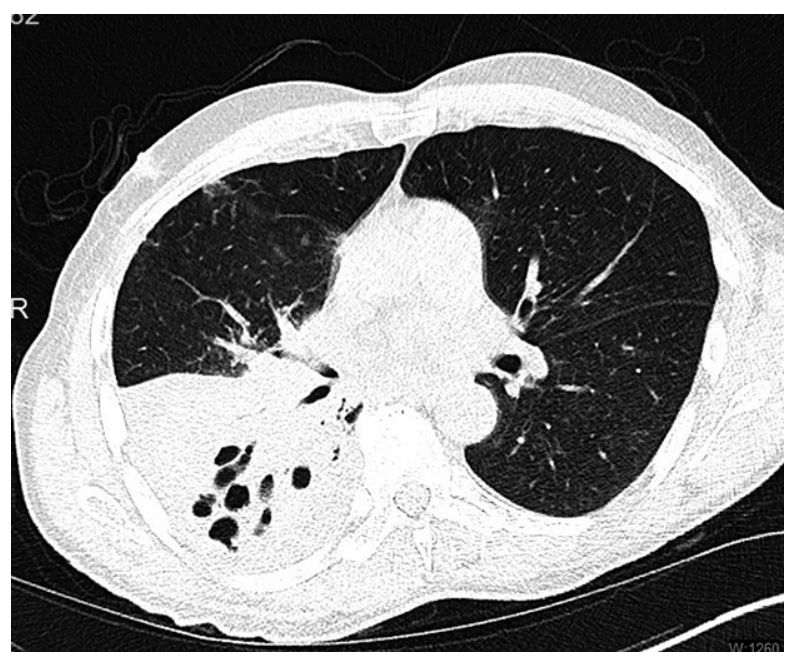

Figure 2. Non-contrast CT lung scan of chest Extensive right-sided airspace opacities with multiple cysts. No air bronchograms

asured. Physical exam was unremarkable except for bilateral rhonchi on auscultation. Chest X-ray (Fig. 1) showed features suggestive of scarring, atelectasis, and cystic changes in the right lung indicating possible bronchiectasis. Non-contrast chest CT (Fig. 2) showed a right-sided pleural effusion and opacity with likely consolidation suspicious of pneumonia.

Sputum and serological testing was then performed that was negative for bacteria and fungi. Screenings for atypical pneumonia microorganisms were negative. Sputum acid-fast bacillus testing and QuantiFERON-TB Gold test were also both found to be negative. Due to non-resolving pneumonia and recent reported weight loss, subsequent bronchoscopy was performed. Biopsies 


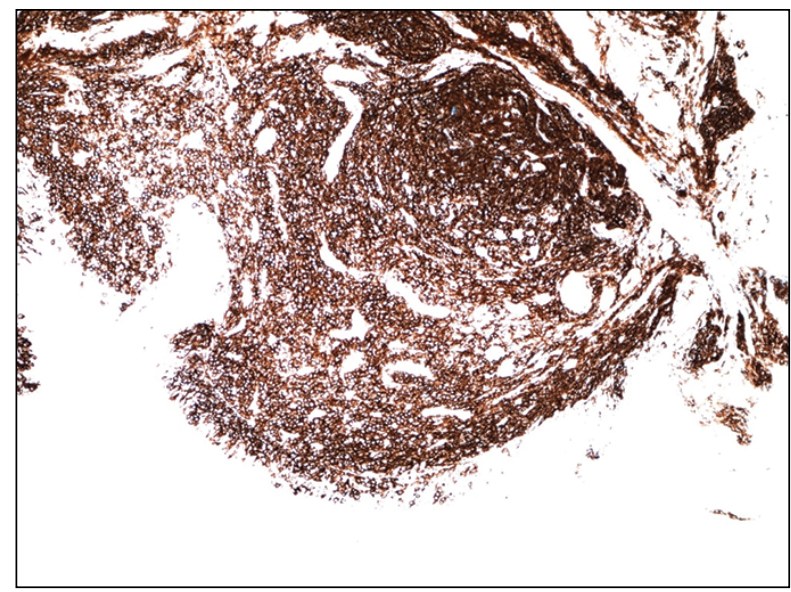

Figure 3. Immunohistochemistry, CD20+, B-cell marker

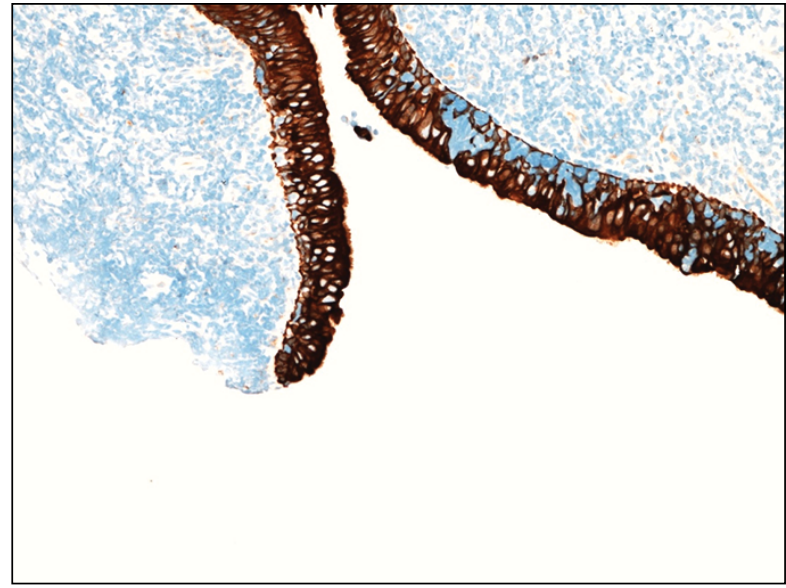

Figure 4. Immunohistochemistry, Bcl-2+. B-cell marginal zone lymphoma marker

taken from the right upper and middle lobe showed MALT extranodal marginal zone lymphoma (MZL) of the bronchial submucosa heavily infiltrated by small-to medium sized lymphoid cells containing round or oval nuclei with dispersed chromatin, inconspicuous nucleoli, and moderate amounts of cytoplasm. Lymphoepithelial lesion (LEL) was focally present, which is frequently associated with MALT lymphoma.

Immunohistochemistry demonstrated neoplastic lymphocytes positive for CD20 (B-cell marker) (Fig. 3) and Bcl-2 (marginal zone lymphoma marker) (Fig. 4). Neoplastic lymphocytes were negative for Bcl-1 (mantle cell lymphoma marker), CD3 and CD5 (T-cell markers), CD10 (acute lymphoblastic leukemia marker), CD23 (IgE receptor antibody), CD43 (T-cell activation marker), and CD56 (natural killer cell marker). These results demonstrate that this patient's diagnosis is a type $2 \mathrm{~B}$-cell lymphoma, consistent with a MALT lymphoma in the right lung. The Ki-67 proliferative index is $10 \%-15 \%$. Anti-Pan Cytokeratin AE1/AE3 fluorophore highlights the focal LEL.

After discovery of PLL further investigation for possible other MALT lesions was performed - ultrasound and computer tomography of the abdomen were unremarkable. Patient was referred to oncology due the extensiveness of the disease and surgery was ruled out. The patient was treated with a chemotherapy regimen of cyclophosphamide, doxorubicin, vincristine, and prednisone (CHOP) and rituximab. After 6 cycles of chemotherapy, positron emission tomography (PET) revealed the patient to be disease free and he was followed on an outpatient basis.

\section{Discussion}

Primary lung lymphoma (PLL) is a rare unilateral or bilateral lung disorder defined as clonal lymphoid proliferation affecting one or both lungs (parenchyma and/or bronchi) in a patient with no detectable extrapulmonary involvement at diagnosis or during the subsequent 3 months [4]. PLL is most frequently a MALT lymphoma: a type of extranodal low-grade B-cell lymphoma characterized by proliferation of clonal marginal zone lymphocytes, which is most traditionally associated with Helicobacter Pylori found in the gastrointestinal tract, specifically the stomach [5]. MALT lymphomas (MALTomas) may also develop due to chronic inflammation or chronic autoimmune diseases e.g. Sjögren's syndrome [2, 6]. PLL presents a clinical challenge, as physical or radiological presentation alone is often not enough to make the diagnosis thus requiring a high index of suspicion, which may help to determine presence of this rare, indolent disease. PLL, due to its non-specific signs and symptoms may be misdiagnosed as pneumonia or a lung tumor. Here we have described a case of a MALT lymphoma located primarily in the lung masking itself as a non-resolving pneumonia.

MALT lymphomas most commonly present non-specifically with $36-50 \%$ of patients being asymptomatic at the time of diagnosis [7, 8]. 58\% of symptomatic patients most commonly reported symptoms of dry cough, dyspnea, chest pain, and hemoptysis; $22 \%$ of symptomatic patients report B type symptoms such as weight loss, fever, and night sweats [7, 9]. B type symptoms overlapped with respiratory manifestations creates a clinical conundrum as was seen in our patient. Initial diagnostic workup in such patients often includes laboratory 
and diagnostic imaging if infectious etiology is suspected. Radiological examinations have shown that non specific patchy opacities or mass like consolidation are seen in up to $68 \%$ of patients [10].

The management algorithm of PLL typically follows that of newly discovered masses with the gold standard being biopsy of the suspicious mass. Sampling techniques include transthoracic needle biopsy, transbronchial biopsy, open thoracotomy and wedge resection by thoracotomy [11]. Our case highlights the use of transbronchial biopsy via bronchoscopy, which is conservative in the spectrum of tissue sampling procedures. Often diagnosis is more challenging and requires more invasive techniques as reported by Cordier et al. and Ferraro et al. in which $89 \%$ and $90 \%$ of patients required an open thoracotomy for definitive diagnosis of PLL [9, 12].

Supplemental diagnostic techniques such as bronchoalveolar lavage (BAL) may also be used to diagnose; however, BAL sensitivity for MALT lymphoma is only $66 \%$ [13]. Immunohistochemistry may also aid in the diagnosis to distinguish MALT lymphoma from other small B-cell non-Hodgkin's lymphomas as it did in this case. B-cell-associated antigens such as CD19, CD20, CD22, and CD79a may be expressed in MALT lymphomas.

Treatment options for primary lung MALT lymphoma treatment are either surgical resection or chemotherapy regimen of CHOP with rituximab. Surgical intervention with adjuvant chemotherapy is preferred to preserve lung function [14]. However, PLL is an indolent disease that does not always require immediate intervention. Most patients show no growth or spread of lymphoma for up to 12 months after diagnosis, and have a median time to progression of 5.6 years [15]. Even without treatment patients have favorable prognosis. The ten-year survival for MZL is 53\% [12] with no difference in survival rates of unilateral versus bilateral disease [10].

\section{Conclusion}

Primary lung lymphomas are a rare occurrence that are difficult to diagnose. They are usually asymptomatic with non-specific clinical manifestations or radiological characteristics. Non-specific symptoms may lead to difficulty in diagnosis. Definitive diagnosis of PLL as a MALT lymphoma is based on histological examination of surgical tissue biopsies. Treatment options for patients like the one presented here could include monitoring progression, surgery, chemotherapy or radiotherapy. There is no current established management protocol. Treatment is instead guided by expert opinion rather than evidence based medicine from randomized clinical trials. If clinical suspicion is high, a full diagnostic workup - possibly including invasive tissue sampling procedures - should be considered to accurately diagnose PLL patients as early as possible.

\section{Conflict of interest}

The authors declare no conflict of interest.

\section{References:}

1. Miller DL, Allen MS. Rare pulmonary neoplasms. Mayo Clin Proc. 1993; 68(5): 492-498, indexed in Pubmed: 8386792.

2. Cadranel J, Wislez M, Antoine M, et al. AIDS-related primary pulmonary lymphoma. Am J Respir Crit Care Med. 1998; 158(4): 1221-1229, doi: 10.1164/ajrccm.158.4.9801057, indexed in Pubmed: 9769285.

3. Isaacson P, Wright DH. Malignant lymphoma of mucosa-associated lymphoid tissue. A distinctive type of B-cell lymphoma. Cancer. 1983; 52(8): 1410-1416, indexed in Pubmed: 6193858.

4. Majid N, Kamal ElB, Oncology B, et al. Primary pulmonary lymphoma: About five cases and literature review. Lung India. 2014; 31(1): 53-55, doi: 10.4103/0970-2113.125909, indexed in Pubmed: 24669084.

5. Parsonnet J, Hansen S, Rodriguez L, et al. Helicobacter pylori infection and gastric lymphoma. N Engl J Med. 1994; 330(18): 1267-1271, doi: 10.1056/NEJM199405053301803, indexed in Pubmed: $\underline{8145781}$.

6. Isaacson PG. Mucosa-associated lymphoid tissue lymphoma. Semin Hematol. . 1999; 36(2): 139-147.

7. Borie R, Wislez M, Thabut G, et al. Clinical characteristics and prognostic factors of pulmonary MALT lymphoma. Eur Respir J. 2009; 34(6): 1408-1416, doi: 10.1183/09031936.00039309, indexed in Pubmed: 19541720.

8. Kurtin PJ, Myers JL, Adlakha H, et al. Pathologic and clinical features of primary pulmonary extranodal marginal zone Bcell lymphoma of MALT type. Am J Surg Pathol. 2001; 25(8): 997-1008, indexed in Pubmed: 11474283.

9. Cordier JF, Chailleux E, Lauque D, et al. Primary pulmonary lymphomas. A clinical study of 70 cases in nonimmunocompromised patients. Chest. 1993; 103(1): 201-208, indexed in Pubmed: $\underline{8417879}$.

10. Parissis H. Forty years literature review of primary lung lymphoma. J Cardiothorac Surg. 2011; 6: 23, doi: 10.1186/17498090-6-23, indexed in Pubmed: 21371331.

11. Wannesson L, Cavalli F, Zucca E. Primary pulmonary lymphoma: current status. Clin Lymphoma Myeloma. 2005; 6(3): 220-227, doi: 10.3816/CLM.2005.n.049, indexed in Pubmed: 16354327.

12. Ferraro P, Trastek VF, Adlakha H, et al. Primary non-Hodgkin's lymphoma of the lung. Ann Thorac Surg. 2000; 69(4): 993-997, indexed in Pubmed: 10800781.

13. Poletti V, Romagna M, Gasponi A, et al. Bronchoalveolar lavage in the diagnosis of low-grade, MALT type, B-cell lymphoma in the lung. Monaldi Arch Chest Dis. 1995; 50(3): 191-194, indexed in Pubmed: 7663488.

14. Lee S, Kim MJ, Kim JS, et al. Consortium for Improving Survival of Lymphoma (CISL) study group. Phase II study of gemcitabine for treatment of patients with advanced stage marginal zone B-cell lymphoma: Consortium for Improving Survival of Lymphoma (CISL) trial. Invest New Drugs. 2010; 28(2): 171-177, doi: 10.1007/s10637-009-9260-6, indexed in Pubmed: 19421710.

15. Troch M, Streubel B, Petkov V, et al. Does MALT lymphoma of the lung require immediate treatment? An analysis of 11 untreated cases with long-term follow-up. Anticancer Res. 2007; 27(5B): 3633-3637, indexed in Pubmed: 17972528. 